\title{
Disturbing the stability of the interface by adopting the phase change temperature gradient to reduce the ice adhesion strength
}

\author{
Tingkun Chen ${ }^{1}$, Jingfu Jin ${ }^{2, *}$, Yingchun Qi $^{2}$, Weijun Tian ${ }^{2}$, Qian Cong,3,**, \\ Kwang-Leong Choy ${ }^{4}$
}

1. Key Laboratory of Bionic Engineering, Ministry of Education, Jilin University, Changchun 130022, P.R.China.

2. College of Biological and Agricultural Engineering, Jilin University, Changchun 130022, P.R.China.

3. State Key Laboratory of Automotive Simulation and Control, Jilin University, Changchun 130022, P.R.China.

4. Institute for Materials Discovery, University College London, London WC1E 7JE, United Kingdom.

\begin{abstract}
Water and its solution are regarded as the low temperature phase change energy released material. The study proposes a novel de-icing model that establishes the phase change temperature gradient at the interface to disturb the contact stability between the substrate surface and the covered ice. During the experiment, the pit with the same size was machined on the surface of aluminum alloy and PMMA which both regarded as the experimental materials and the ethanol solutions with the different freezing points were filled in the pits. The ice adhesion strength on the substrate was repeatedly tested under the different temperatures. The experimental results shown that the ice adhesion
\end{abstract}

${ }^{*}$ Corresponding author: Jingfu Jin.

${ }^{* *}$ Corresponding author: Qian Cong. E-mail address: jinjingfu@jilu.edu.cn (Jingfu Jin), congqian@jilu.edu.cn (Qian Cong) Fax number: +86-431-85095253 (Jingfu Jin), +86-431-85095575-888 (Qian Cong) Add: No. 5988 Renmin Street, Changchun, 130025, China 
strength on the sample with the phase change temperature gradient was $0 \mathrm{~N}$ in contrast to the ice adhesion strength on the smooth sample. That meant that the proposed deicing model had the excellent de-icing capability. Owing to the constraint of the rigid boundaries of the pit, the phase change energy released by the solution filled in the pits in the different locations hit on the elastic interface between the covered ice and substrate, at intervals. This would cause the deformation of the interface and the accreted ice is always subjected to the upward impingement load. The contact stability of the interface is affected and the accumulated ice is separated from the sample surface. Adopting the water solutions to release the release energy at low temperatures could enhance the active de-icing characteristic of the anti-icing components, also provide a novel method to develop the new de-icing methods and the de-icing materials.

Keywords: low-temperature energy release; aqueous solution; phase change; temperature gradient; deformed interface; ice adhesion strength

\section{Introduction}

Ice, frost and wet-snow adhered to the exposed structures and equipment is a common natural phenomenon, not only lead to many catastrophic consequences for human activity but also cause socio-economic loss in cold-climate regions. For examples, in aviation, ice accumulation on surface of the airplane during flights, changes the effective shape of plane, and alters the aerodynamic parameters owing to the air flow over iced parts, and has notably negative influence on the operational reliability (Boinovich et al., 2013; Caliskan et al., 2013; Gent et al., 2000). It is well- 
known that wind energy is one of the most promising energy owing to the renewable and clean characteristics of wind energy, and the installed capacity of wind turbines increases per year gradually (Dalili et al., 2009). The wind turbines are frequently built in cold regions or high altitudes and often operates during the icing conditions. Ice accretion on the wind turbines affects the stability of the equipment, safe operation, causes the mechanical failures and decreases the productivity due to the change in aerodynamic parameters (Hu et al., 2017; Pérez et al., 2016; Parent et al., 2011). Another field significantly influenced by the ice accretion is the power transmission. The accreted ice on the structures, like the tower and line, increases their load, causes many severe incidents and pose a great hazard to people's lives, including the 2008 icing disaster in China (Drage et al., 2008; Ruan et al., 2016). The disaster has caused the direct economic loss up to more than 150 billion RMB ( 23 billion $\$$ ) and leaded to spending 39 billion RMB ( $\sim 6$ billion $\$$ ) reconstructing the damaged power system (Lv et al., 2014). In addition, the phenomenon of ice accumulation on the exposed surface has significantly influenced many industries, such as the transportation field, telecommunication networks, refrigeration, solar photovoltaic cells and so on (Boinovich et al., 2013; Bewilogua et al., 2009).

Water adhered to the exposed surface and solidified into the ice can be regarded as an adhesive during the freezing process. Hence, the researchers are devoted to developing new anti-icing methods to reduce the amount of water adhesion or the ice adhesion strength to mitigate the hazards of the ice accumulation. More than 30 antiicing or de-icing methods has been formed and can be classified into three categories: 
mechanical ways to remove the ice accumulation directly, heating de-icing methods to delay the freezing time or melt the covered ice, and chemical agents changed the phase transition temperature of water to delay the freezing time (Rashid et al., 2016; Zhang et al., 2015). However, the conventional de-icing methods have many shortcomings during the actual use, for example, mechanical scraping requirement a large amount of cost, the thermal method consuming much energy with the poor economy, and the chemical agents producing pollution and corrosion the metal parts and so on (Liu et al., 2016; Ozbay et al., 2015,2016). This motivates researchers and engineers to optimize the existing methods to extenuate these drawbacks.

Recently, since the self-cleaning characteristics of the lotus leaf discovered, many studies focus on fabrication the functional materials with icephobic property and improvement the wettability of the material surface to achieve the hydrophobicity. And the superhydrophobic surfaces are regarded as a promising way to clean the accreted ice on the exposed surface owing to their extraordinary water-repellency or reduce the ice adhesion under the experiment conditions (Boreyko et al., 2013; Parent et al., 2011; Kim et al., 2012; Mobaraken et al., 2013). However, there are many literatures that have reported the superhydrophobic surfaces with poor durability owing to experiencing multiple freeze-thaw cycles or subjecting in high humidity conditions (Bharathidasan et al., 2014; Chen et al., 2012; Dodiuk et al., 2012; Farhadi et al., 2011; Jung et al., 2011; Lazauskas et al., 2013; Kulinich et al., 2011; Nosonovskv et al., 2012). The superhydrophobic coating is weakened by various factors, including surface microstructure damage, surface contamination and et al (Boiniovivh et al., 2012; 
Farhadi et al., 2011; Kulinich et al., 2011; Lazauskas et al., 2013; Ozbay et al., 2016; Oberli et al., 2010; Mahadik et al., 2013; Shin et al., 2011; Wang et al., 2015; Zhu et al., 2014). Kulinich and co-workers (Kulinich and Farhadi, 2011) have investigated that the microstructure of the superhydrophobic surface gradually destroyed after the icing and de-icing experiment, and other researchers consider that the damaged microstructure of the superhydrophobic surfaces is caused by the compressive stress generated during ice expansion (Lazauskas et al., 2013; Oberli et al., 2014). In addition, some researchers deem that water attached to the superhydrophobic surfaces for a long time induces the reaction, and results in the water molecular easily penetration into the interface between the substrate and coating (Mahadik et al., 2013). In summary, if superhydrophobic coatings are to be applied into the engineering, more efforts are needed to be done on improvement the durability of the superhydrophobic surfaces.

Hence, to mitigate the cost of the conventional anti-icing methods and improve the durability of de-icing on superhydrophobic surfaces, many researchers have been carrying out the study about the mechanism of the icing on the exposed surface. Bobinski et al. (2014) investigated the freezing droplet, various droplet impingement, and deformation on the different physic-chemical properties by using high-speed camera. Zhang et al. (2016) studied the effect of surface energy of substrates with the static angle varying from $5^{\circ}$ to $110^{\circ}$ on freezing temperature of water by adopting the infrared-visible sum frequency generation spectroscopy. Alizadeh et al. (2012) proposed an analysis to explain the dynamic of water frozen into the ice on surfaces with different wettabilities, and demonstrated the ice nucleation are postponed by the 
chemistry and texture of the substrate surface under the condition of low humidity tests. Tavakoli et al. (2015) experimentally investigated that many parameters, such as the surface roughness, drop volume et al, affect the spreading ability of the water droplets on the cooling hydrophobic surfaces.

Presently, the energy consumption is rapid increasing and the energy crisis is becoming more and more obvious. In order to solve the contradiction between energy supply and demand in time and space, many researchers have been exploring the technologies and materials for thermal energy storage. And the phase change stored energy material has been applied in some engineering fields, such as solar energy storage system and air conditioning energy storage system and so on. It is well known that water and aqueous solution are regarded as the phase change stored energy material. And the water freezes into ice and the energy is released under low temperature conditions. The released energy is showed as a significant increases in volume and inner temperature (Alizadeh et al., 2012; Tavakoli et al., 2015). If there are boundary constraints around, the released energy could also be demonstrated by the frost heaving force, such as the bursting of aluminum water tanks in winter. Meanwhile, the released energy has caused many damages which will lead to reducing the service life and operational safety of the engineering structures (McDonald et al., 2017; Rashid et al., 2016; Sultana et al., 2018), and bringing inconvenience to our daily life, such as the emerged cracks of the reservoirs, breakwaters, retaining walls and other marine structures, the rupture of water transmission pipe, etc. Hence, why not rationally adopt the energy storage or release characteristics of phase change stored energy material to 
remove the accumulated ice on the material surface.

According to the phenomenon of the multi points blasting in the mine filed, the present paper puts forward an anti-icing model: one that utilizes the characteristics of phase change energy release of water and its aqueous solution to remove the accumulated ice on the material surface, and the aqueous solutions with different freezing points are buried at different locations at the contact interface to make a gradient of the phase change temperature. During the model, the phase change energy released at different times could be regarded as the active power source to influence or damage the contact stability of the interface. Namely, many impingement forces generated at different times and different positions directly act on the interface. This principles is similar to the phenomenon of blasting in different areas of the structure to influence the strength and connect stability, such as mining and stone excavation, et al. And the ice adhesion strength will be decreased, the accreted ice will be easily removed and the de-icing cost will be reduced.

\section{Materials and methods}

\subsection{De-icing model and principle}

\subsubsection{Freezing characteristics of droplet}

It is well-known that the volume and height of the water droplet on the surface of the material must increase after experiencing phase transformation. Meanwhile, the phase transformation energy can be releases in a short time. For example, the freezing process of 5 microliters of water on the surface of 6061 aluminum alloy was observed, 
and the characteristic parameters were collected during the solidification process, as shown in Fig 1 . The inner temperature of $5 \mu$ of water droplets suddenly increased from $-14{ }^{\circ} \mathrm{C}$ to $-12.4{ }^{\circ} \mathrm{C}$ within 7 seconds.

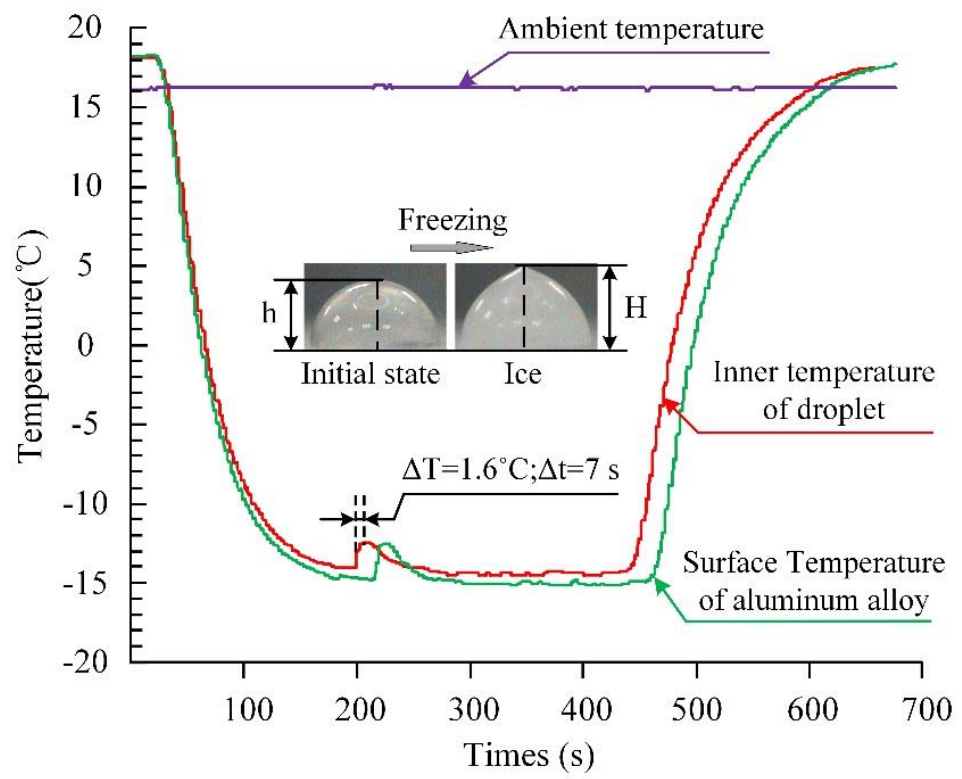

Fig 1. The collected temperature curves during the freezing process

The released phase change energy of water could be demonstrated by the increase of the inner temperature and of the volume of the droplet (as shown in Fig 1.), and the frost heaving force acting on the surrounding constraints, such as the bursting of the water tank in winter.

\subsubsection{Active de-icing model}

This study proposes an active de-icing model that adopts the phase change energy generated at different locations and different times to act on the icing interface, affect the stability of the contact interface to reduce the ice adhesion strength. The diagram of the de-icing model is shown in Fig 2. The active de-icing model consists of the film, the substrate and the water solution which is regarded as the low temperature phase 
change material. The substrate has many pits filled with water solutions that have different freezing points, and the surface of the substrate is covered by a thin film with elasticity, such as the biaxially oriented polypropylene (bopp). Therefore, there are two kinds of difference in phase transition time during the model: one is the phase change time difference between the solution filled in the pit and water attached the surface of film owing to the thermal conductivity of substrate and other factors. The solution filled in the pit also includes water and the solution with lower freezing point than the water. And the other is the phase change time difference between the different solutions in the pits.

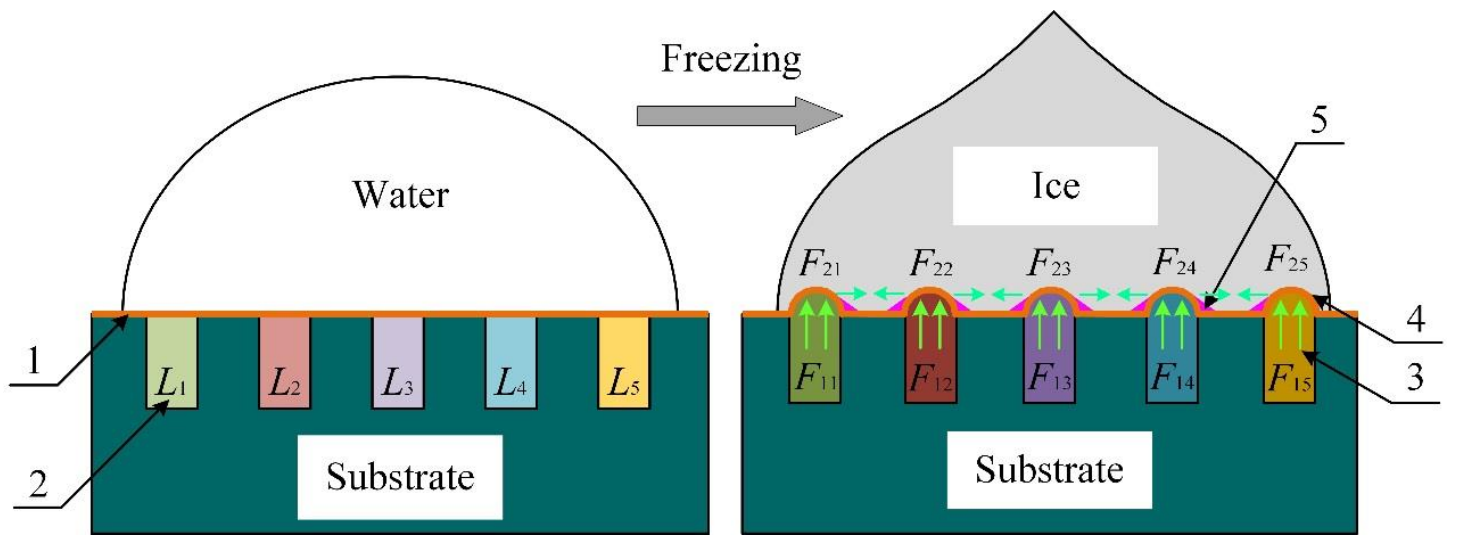

1. Flat film; 2. Water solution; 3. Ice filled in the pits; 4 . Deformed film; the arrow indicates the frost heaving force; $L_{1}, L_{2}, L_{3}, L_{4}, L_{5}$, respectively represent solutions with different freezing points; 5 . Contact defects.

Fig 2. Schematic of the proposed de-icing model.

At low temperature, the water attached on the surface would freeze firstly and the contact stability is gradually stable. Owing to the establishment of phase change temperature gradient in the de-icing model, the solutions begin to solidify into ice orderly as the cooling continues, and the volume of solutions would increase. The frost 
heaving forces generated at different times and in different locations of the interface directly act on the contact interface. In other words, many forces impinge on the contact interface, and seriously affect the contact stability. And the height of solution after freezing into ice is higher than the initial height due to the constraints of the pit boundaries. These would cause deformation of the elastic thin interface and the morphology of the contact interface that looks like the rolling hills. The ice adhesion strength would decrease, and the accumulated ice would be easy to remove.

\subsection{Materials}

The ice adhesion strength was adopted to demonstrate the effectiveness of the proposed de-icing model, the cup method was applied for ice fabrication during the experiment, as shown in Fig 3. The aluminum cup had an inner diameter $32 \mathrm{~mm}$. In order to exclude the influence of the thermal conductivity of the sample on the effect of the proposed model, the tests took 6061 aluminum alloy and polymethyl methacrylate (pmma) as samples to represent good and poor thermal conductivity materials respectively. The thermal conductivities of 6061 aluminum alloy and pmma are $237 \mathrm{~W} / \mathrm{m} \cdot \mathrm{k}$ and $0.2 \mathrm{~W} / \mathrm{m} \cdot \mathrm{k}$ respectively.

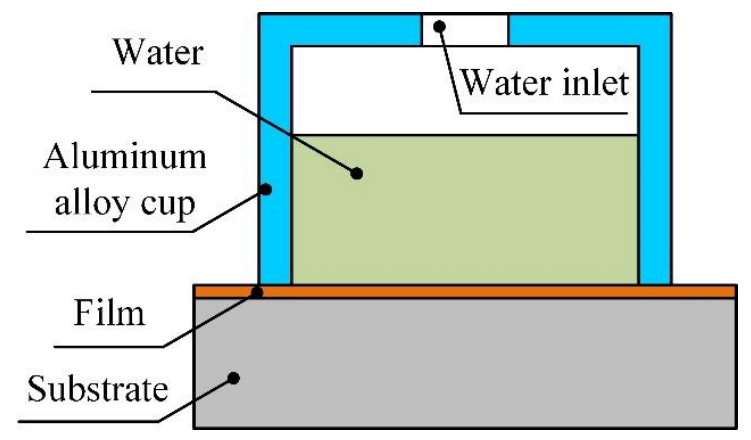

Fig 3. Cup method to fabrication ice. 
During the experiments, all samples were $50 \mathrm{~mm} \times 50 \mathrm{~mm} \times 5 \mathrm{~mm}(\mathrm{~L} \times \mathrm{W} \times \mathrm{H})$ in size. In the central area of the sample surface, there were 5 rows and 5 columns of pits with diameters of $3 \mathrm{~mm}$ and depth $4 \mathrm{~mm}$, and the distance between the adjacent pits was $4.55 \mathrm{~mm}$. The pit was machined by using the milling method and filled with different mass concentration of ethanol solution. The surface of the substrate was covered with the biaxially oriented polypropylene (bopp) having a thickness of 0.053 $\mathrm{mm}$.

\subsection{Characteristics of freezing medium}

The proposed de-icing model utilizes the established phase change temperature gradient and takes the frost heaving force generated from the solutions filled in the pits at different locations to act on the interface and influence the contact stability. The ice adhesion strength would be reduced. Namely, there were many solutions with different freezing point below the water in a large number of pits of the sample. Hence, the different mass concentrations of alcohol solution were applied to fill the pits, so there are multiple phase change temperature differences in the model. The freezing temperatures of alcohol solutions with mass concentrations of $0 \%$ (water), $4.5 \%, 9 \%$, $13 \%, 16.5 \%$ are shown in Table 1.

Table 1 Freezing temperature of alcohol solutions with different mass concentrations.

\begin{tabular}{cccccc}
\hline Mass concentration & $0 \%$ & $4.5 \%$ & $9 \%$ & $13 \%$ & $16.5 \%$ \\
\hline Freezing point $\left({ }^{\circ} \mathrm{C}\right)$ & 0 & -2 & -4 & -6 & -8 \\
\hline
\end{tabular}

\subsection{Experimental details}


During the experiments, five kinds of ethanol solutions with different mass concentration were filled in the pits, resulting in a gradient of phase change temperature at the interface, as shown in Fig. 4. The temperature difference between the adjacent solutions was $-2{ }^{\circ} \mathrm{C}$.

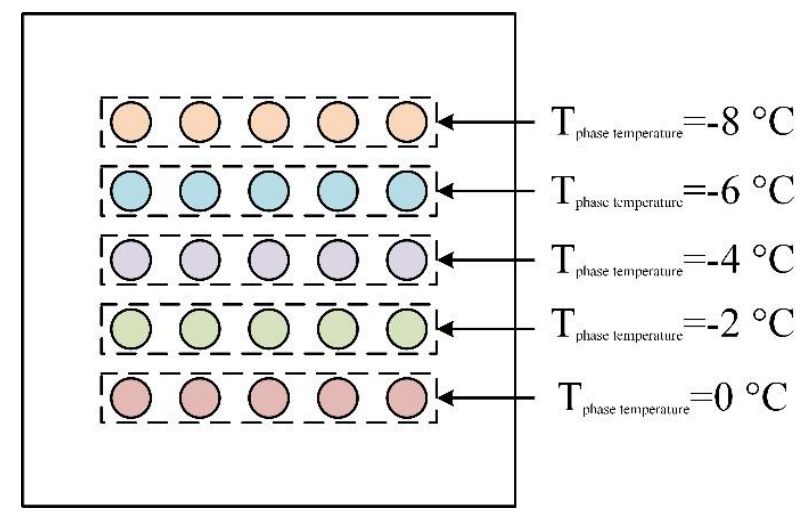

Fig 4. Phase change temperature gradient on the interface.

The total de-icing model was placed in the climate chamber that controlled the experiment temperature at $-10{ }^{\circ} \mathrm{C},-15{ }^{\circ} \mathrm{C},-20{ }^{\circ} \mathrm{C},-25^{\circ} \mathrm{C}$, respectively, and the refrigeration process lasted 1 hour. The temperature control accuracy of the climate chamber was $\pm 0.01{ }^{\circ} \mathrm{C}$. During the process of water attachment and ice formation, water could be regarded as an adhesive agent and solidified into ice at low temperature. At the same time, the ice adhesion strength was formed between the ice and substrate. Therefore, the experimental apparatus was designed according to the ASTM-D3528 (2008) standard. As shown in Fig 5, the test device consisted of draft gauge, worm gear, fixed pulley and other components. Additionally, the base and the sample holder were made of the insulating material to decrease the effect of heat transfer on the interface. 


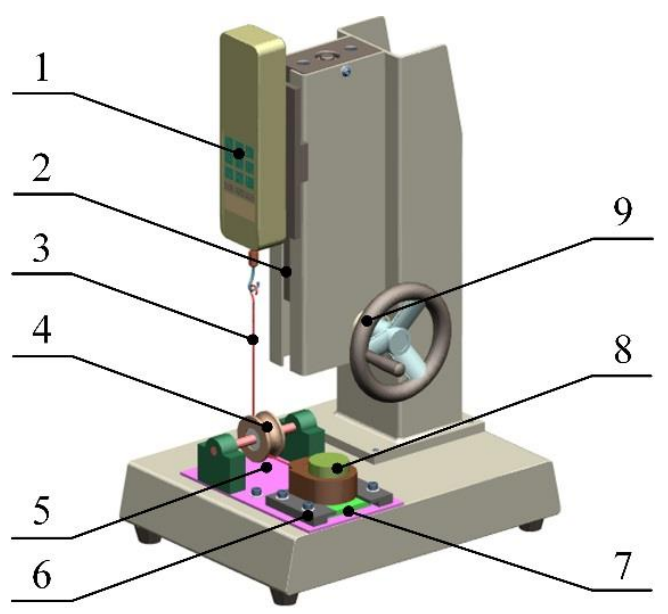

1. Draft gauge; 2. Worm gear; 3. Wire rope; 4. Fixed pulley; 5. Base; 6. Sample holder; 7. Sample; 8. Aluminum cup; 9. Hand wheel.

Fig 5. The experimental apparatus

During the test, the draft gauge could be moved up and down to remove the accumulated ice from the surface by rotating the hand wheel to drive the worm gear. At the same time, the ice adhesion strength was measured and recorded during the movement of the draft gauge. In this paper, the maximum force of the record was defined as the ice adhesion strength during the peeling process and used to evaluate the de-icing effect of the proposed de-icing model, and the unit is $N$. Compared with other assessments, this method is more intuitive, simple and consistent with the reality. The precision of the draft gauge is $0.01 \mathrm{~N}$.

\section{Results}

\subsection{Ice adhesion strength}

The ice adhesion strength on the surface of the de-icing model was measured during the environment with different temperatures, and the ice adhesion strength on 
the substrate without the pit was regarded as the reference and evaluated the de-icing efficiency of the de-icing model. During the experiments, the average experimental results were taken as the ice adhesion strength of the sample in question. The experimental results were shown in Fig 6.

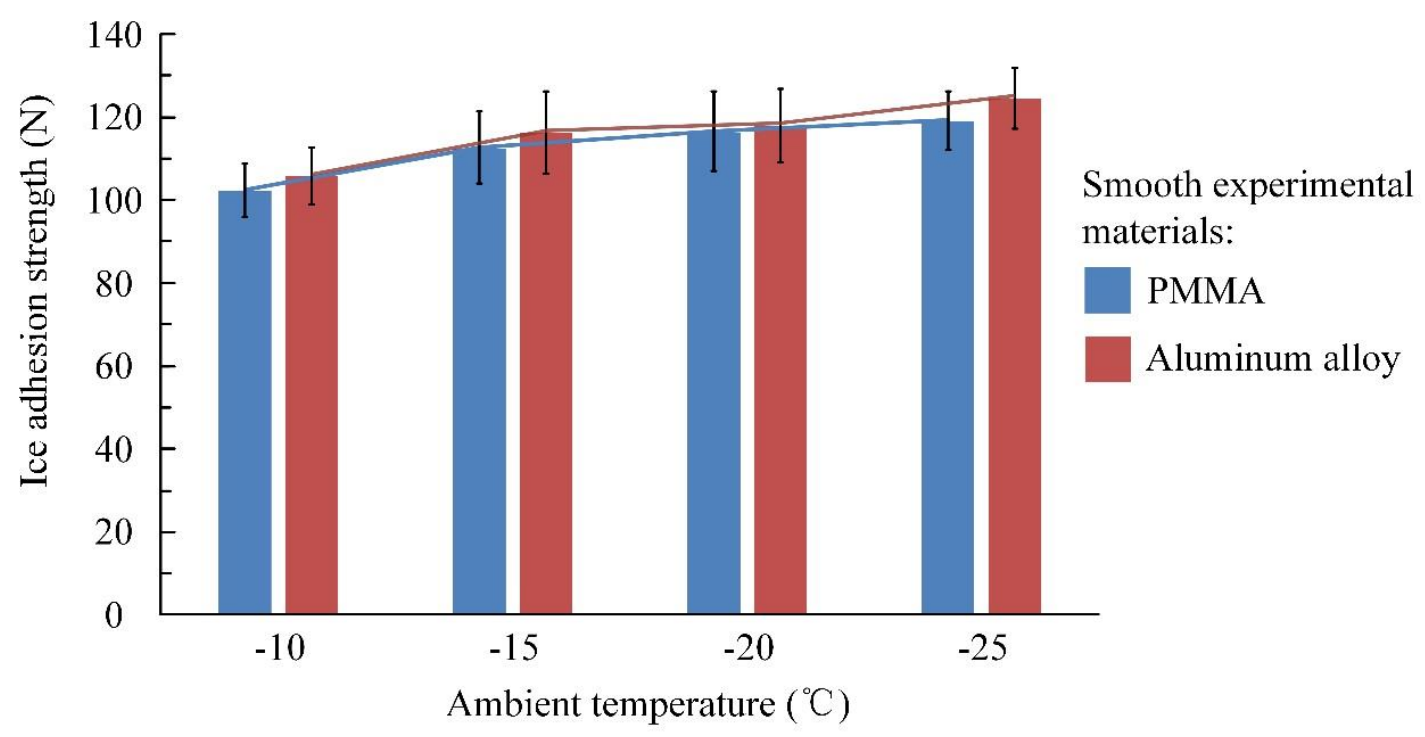

Fig 6. Shear ice adhesion strength.

After repeated tests, the ice adhesion strength on the surface of the de-icing model with the phase change temperature gradient was always $0 \mathrm{~N}$, and the reduction rate of the ice adhesion strength on the de-icing model surface reached $100 \%$. The proposed de-icing model demonstrated a significant de-icing effect. The ice adhesion strength of the smooth aluminum alloy was higher than that of the smooth PMMA surface, and the ice adhesion strength of both surfaces increased as the ambient temperatures decreased. Meanwhile, the growth rate of the ice adhesion strength gradually decreased.

\subsection{Interface topography after testing}

Since the elastic film covered the pits on the sample surface, the aqueous solution 
in the pit released the stored energy during the process of the phase change. The released energy would directly impinge on the contact interface, so that the covered film and the icing interface would be deformed, as shown in Fig 7 (a). The morphology of the elastic film on the substrate was markedly changed, and it looked like the undulating hills, as shown in fig 7 (a).

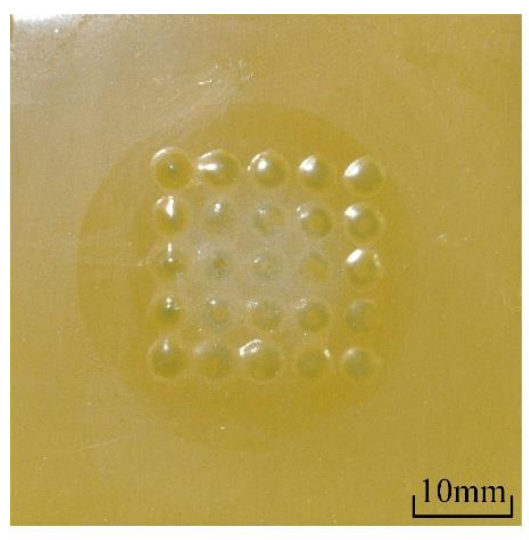

(a) Deformed covered film

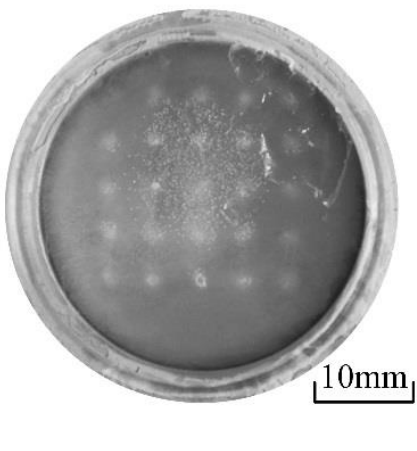

(b) Deformed icing interface

Fig. 7. Deformed film and icing interface after freezing and peeling off.

Additionally, there were many stress concentration points on the icing interface caused by the phase change energy released by the filling solution in the pits. These could be distinguished and seen from the white dots with ordered distribution in fig 7 (b). Meanwhile, many tiny ice crystals appeared at the center of the sample and icing interface, as shown in fig 7. During the experiments, when the ambient temperature was lower, the phenomenon of the appearance of the ice crystals was more obvious. Namely, it meant that when the ambient temperature was $-25^{\circ} \mathrm{C}$, the time of ice peeling on the substrate was earlier than at $-10{ }^{\circ} \mathrm{C}$.

\subsection{Freezing characteristics of the solution}

The proposed de-icing model established the phase change temperature gradient 
on the substrate surface, and utilized the energy released at different locations and at different times to impinge the contact interface between ice and substrate. Hence, in order to better understand the action mechanism of the de-icing model, it is necessary to understand the phase change time of different freezing media in different temperature surroundings. The phase change time of the different mass concentration solutions filled in the pits of the PMMA and aluminum alloy was tested. Table 2 shown the phase change time of the different mass concentration solution used during the environment with $-10{ }^{\circ} \mathrm{C},-15^{\circ} \mathrm{C},-20{ }^{\circ} \mathrm{C},-25^{\circ} \mathrm{C}$, respectively.

Table 2 Phase change time of different mass concentration of ethanol solution at the different ambient temperatures (s).

\begin{tabular}{ccccccc}
\hline \multirow{2}{*}{$\begin{array}{c}\text { Ambient } \\
\text { temperature }\left({ }^{\circ} \mathrm{C}\right)\end{array}$} & Substrate & \multicolumn{5}{c}{ Mass concentration } \\
\cline { 2 - 7 }-10 & $\begin{array}{c}\text { Aluminum } \\
\text { alloy }\end{array}$ & 905 & 1709 & 2551 & N/A & N/A \\
\cline { 2 - 7 } & PMMA & 1697 & 2672 & 3316 & N/A & N/A \\
\hline-15 & $\begin{array}{c}\text { Aluminum } \\
\text { alloy }\end{array}$ & 581 & 890 & 1080 & 1352 & 1550 \\
\cline { 2 - 7 } & PMMA & 789 & 1226 & 1323 & 1490 & 1527 \\
\hline-20 & $\begin{array}{c}\text { Aluminum } \\
\text { alloy }\end{array}$ & 412 & 523 & 602 & 738 & 806 \\
\cline { 2 - 7 } & PMMA & 488 & 598 & 649 & 745 & 939 \\
\hline & $\begin{array}{c}\text { Aluminum } \\
\text { alloy }\end{array}$ & 297 & 329 & 365 & 510 & 596 \\
\cline { 2 - 7 } & PMMA & 366 & 442 & 548 & 645 & 781.5 \\
\hline
\end{tabular}

As shown in Table 2, the phase change time of the different mass concentration solution would be prolonged with the increase of the mass concentration of ethanol. 
The lower the ambient temperature was, the earlier the phase change of the solution embedded in the sample was. Due to the small temperature difference between the ambient temperature and the phase change temperatures of ethanol solution with mass concentration of $13 \%$ and $16.5 \%$, the two solutions would take a long time to undergo the phase change. And the phase change time exceeded $1 \mathrm{~h}$, so they were ineffective during the two temperatures. Owing to the difference in the thermal conductivity between the aluminum alloy and PMMA, the phase change time of the solution buried in the pit of the aluminum alloy sample was changed earlier than that embedded in the PMMA.

Hence, the energy stored in the solution filled in the pits would act on the interface at different times, which caused an orderly impingement on the interface, such as the blasting phenomenon. As a result, the stability of the contact interface would be destroyed and the deformation of the membrane was caused.

\section{Discussion}

Based on the experimental results, whether the substrate material was aluminum alloy or PMMA, the proposed de-icing model had the excellent de-icing capabilities through designing the phase change temperature gradient on the substrate surface. During the experiments, the solutions with different freezing points were filled in the pits of the substrate. Due to the difference in mass concentration, the phase change time

of the ethanol solution in the pits was also different. During the experiment, the sequence of icing of the different mass centration of ethanol solution was $0 \%, 4.5 \%$, 
$9 \%, 13 \%, 16.5 \%$. As mentioned above, the shown form of the phase change energy were the increase of internal temperature, the expansion of volume and the frost heaving force acting on the surrounding constraints.

Under the low temperature, due to the thermal conductivity of the material, the water located on the substrate surface would freeze earlier than the water solution filled in the pit. The connection between the substrate and the ice frozen by the surface attached water gradually became stable, as shown in Fig 8 (b). When the ethanol solution with low mass concentration froze into the ice, the release of phase energy and the increase in volume would take place in a short time. As the solution filled in the closed pit, the rigid wall and the upper elastic interface formed the closed space. The elastic interface was impinged by the phase change energy, significantly. Hence, the elastic film covered the substrate was deformed and the point of impingement force loading appeared orderly at the icing interface. This could be seen from the Fig 7 (b) and Fig 8 (c). The frost heaving force generated during the phase change process would directly act on the icing interface and cause the stress concentration area at the icing interface, which would seriously affect the stability of the interface between ice and substrate. This would have a great influence on the ice adhesion strength. 


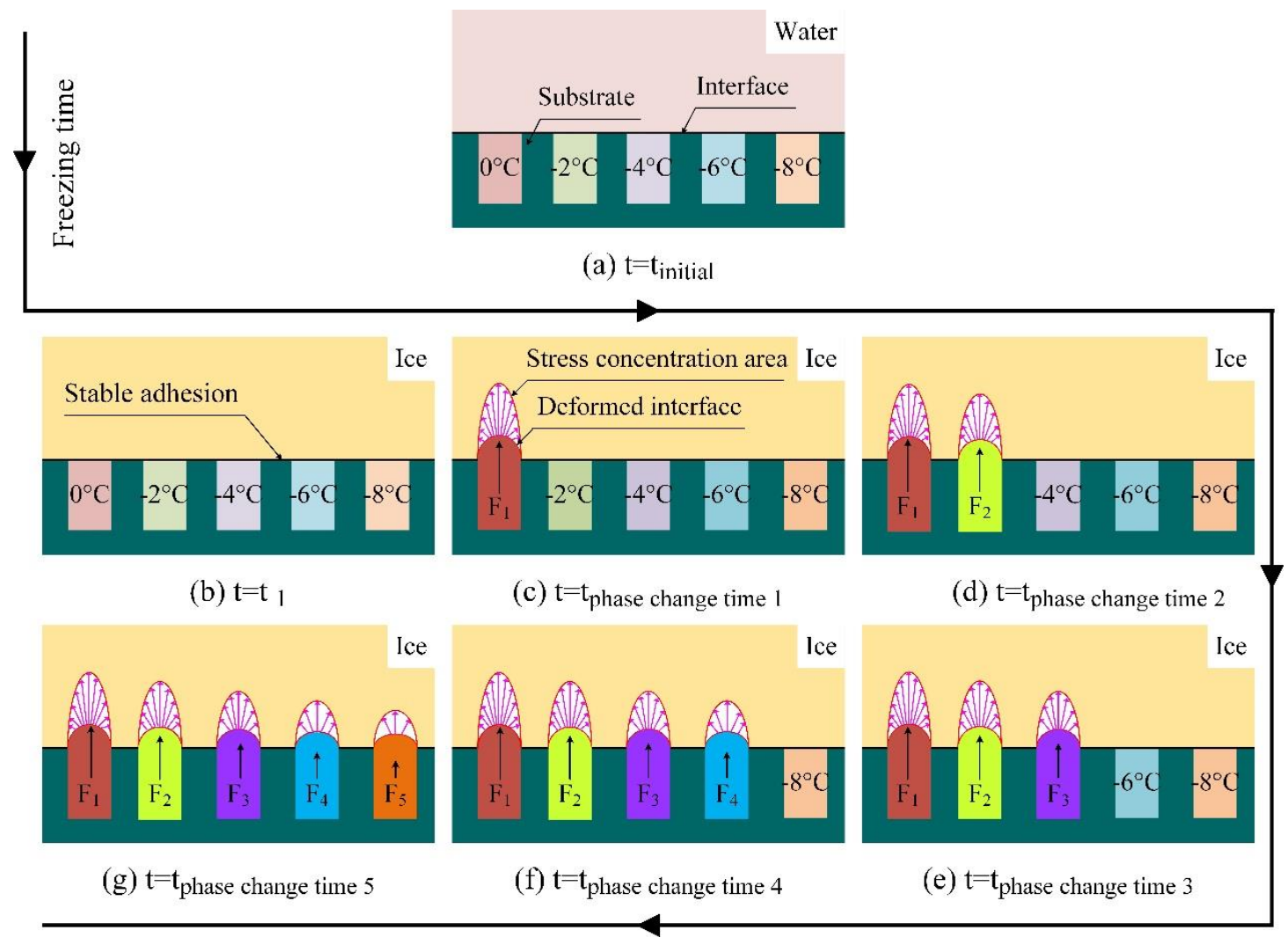

Fig 8. Effect of phase change temperature gradient on the stability of the interface.

The intensity of stress concentration increased with the number of red arrows, and the magnitude of the frost heaving force was represented by the length of the black arrow.

With the continuation of the cooling, the solution filled in the pit would continue to undergo the phase change and freeze into ice. The different mass concentration of the ethanol solution undergone the phase change at different time, as shown in Fig 8. Therefore, the phase change energy could be released at different time. The phase change energy released at different locations and time would act on the flexible film and the icing interface. The volume expansion and frost heaving force generated from the phase change, like the locating blasting in mine, constantly hit the icing interface and destroyed the stability of the interface. This was fatal to the formed stable connection between the covered ice and the substrate surface. Meanwhile, this would 
cause the stress concentration at the icing interface, as shown in Fig 8.

The continuously released phase change energy could always impinge on the contact interface and make the accumulated ice on the surface undergo an upward expansion load. The phenomenon would affect and change the stability of the interface, significantly. As a result, the covered ice would be stripped from the substrate surface, and the white force dots appeared on the icing interface, as shown in Fig 7. In other words, the ice adhesion strength on the substrate surface would be $0 \mathrm{~N}$, the accreted ice was cleanly removed from the sample surface. Additionally, the lower the mass concentration of ethanol solution was, the higher the released energy was, the earlier the phase change took place. When the ambient temperature was lower, the earlier the phase change of the ethanol solution occurred, the sooner the impingement on the interface would be, as shown in table 2 . Compared with the environment at $-10{ }^{\circ} \mathrm{C}$, the earlier the accreted ice was peeled off from the surface of the sample during the environment with $-25^{\circ} \mathrm{C}$, the probability of frost crystals appeared in the central area of the sample surface was higher.

\section{Conclusion}

In summary, combined with the low temperature phase change energy release characteristics of water and its aqueous solution, the proposed de-icing model interfered the stability of the interface by establishing the phase change temperature gradient, and achieved the purpose of reducing the ice adhesion strength. Thus, it was easy to remove the accreted ice on the substrate surface. During the test, the 6061 aluminum alloy and 
PMMA were adopted as the materials with good thermal conductivity and poor thermal conductivity, respectively, and were used as the substrate during the proposed de-icing model. Different mass concentration of ethanol solution were filled in the pits of the substrate surface, and the gradient of the phase change temperature was established at the interface. The ice was completely peeled off from the aluminum alloy or polymethyl methacrylate (PMMA) substrates having pits filled with different mass concentration of ethanol solution, and the ice adhesion strength was 0 . The experiments demonstrated that the proposed de-icing model had the significant de-icing effect.

During the environment with low temperature, water attached on the sample surface freezes into ice firstly due to the thermal conductivity of the substrate, and the ice on the surface tends to become more stable. Then the solution with different freezing points filled in the pits successively undergoes the phase change. Due to the elastic properties of the upper interface of the pit, the energy released by the filled solution during the phase change process could continuously act on the elastic interface. This means that the elastic interface is constantly subjected to the impingement of the phase change energy released at different times and locations. This would cause the deformation of the elastic film and make the stress concentration areas on the icing interface. Hence, the ice that has become stable on the interface has been subjecting to the interval phase change impingement of the filling solutions and the adhesion stability of ice is affected seriously. Moreover, the accreted ice is always subjected to the upward and interval phase change impingement. In short, this would lead to the accumulated ice peeled off from the material surface and make the anti-icing components have the 
active de-icing performance.

Based on the experimental results, this study provides a novel de-icing method. Compared with the conventional de-icing methods and ice-phobic coatings, the proposed de-icing model has many outstanding advantages, such as the active de-icing characteristic, high de-icing efficiency, low fabrication cost, without pollution and good durability, etc. Meanwhile, we could get an inspiration to develop the de-icing method which could be applied on the chassis of the high-speed rail and fabricate the honeycomb polymer membrane which could be stuck onto the surface of the aircraft wing and fan blade, such as the microencapsulated phase change material.

\section{Acknowledgements}

This work was supported by the international exchanges scheme between the Royal Society and the NSFC [Grant No. 51711530236].

\section{References}

Alizadeh, A., Yamada, M., Li, R., et al., 2012. Dynamics of ice nucleation on water repellent surfaces. Langmuir. 28(6), 3180-3186.

Boinovich, L.B., Emelyanenko, A.M., Ivanov, V.K., et al., 2013. Durable Icephobic coating for stainless steel. ACS Appl. Mater. Interfaces. 5(7), 2549-2554.

Boinovich, L.B., Emelyanenko, A.M., 2013. Anti-icing potential of superhydrophobic coatings. Mendeleev Commun. 23(1), 3-10.

Boiniovivh, L.B., Emelyanenko, A.M., Pashinin, A.S., et al., 2012. Origins of 
thermodynamically stable superhydrophobicity of boron nitride nanotubes coatings. Langmuir. 28(2), 1206-1216.

Bewilogua, K., Bräuer, G., Dietz, A., et al., 2009. Surface technology for automotive engineering. CIRP annals - Manuf. Technol. 58 (2), 608-627.

Boreyko, J.B., Collier, C.P., 2013. Delayed frost growth on jumping-drop superhydrophobic surfaces. ACS Nano. 7(2), 1618-1627.

Bharathidasan, T., Kumar, S.V., Bobji, M.S., et al., 2014. Effect of wettability and surface roughness on ice-adhesion strength of hydrophilic, hydrophobic and superhydrophobic surfaces. Appl. Surf. Sci. 314, 241-250.

Bobinski, T., Sobieraj, G., Gumowski, K., et al., 2014. Droplet impact in icing conditions-the influence of ambient air humidity. Arch. Mech. 66(2), 127-142.

Caliskan, F., Hajiyev, V., 2013. A review of in-flight detection and identification of aircraft icing and reconfigurable control. Prog. Aerosp. Sci. 60, 12-34.

Chen, J., Liu, J., He, M., et al., 2012. Superhydrophobic surfaces cannot reduce ice adhesion. Appl. Phys. Lett. 101, 111603.

Dalili, N., Edrisy, A., Carriveau, R., 2009. A review of surface engineering issues critical to wind turbine performance. Renew Sust. Energ. Rev. 13(2), 428-438.

Drage, M.A., Hauge, G., 2008. Atmospheric icing in a coastal mountainous terrain. Measurements and numerical simulations, a case study. Cold Reg. Sci. Tech. 53(2), $150-161$

Dodiuk, H., Kenig, S., Dotan, A., 2012. Do self-cleaning surfaces repel ice? J. Adhes. Sci. Technol. 26, 701-714. 
Farhadi, S., Farzaneh, M., Kulinich, S.A., 2011. Anti-icing performance of superhydrophobic surfaces. Appl. Surf. Sci. 257, 6264-6269.

Gent, R.W., Dart, N.P., Cansdale, J.T., 2000. Aircraft icing. Philos. Trans. R. Soc. AMath. Phys. 358(1776), 2873-2911.

Hu, L.Q., Zhu, X.C., Hu, C.X., 2017. Wind turbines ice distribution and load response under icing conditions. Renew. Energy. 113, 608-619.

Jung, S., Dorrestijn, M., Raps, D., et al., 2011. Are superhydrophobic surfaces best for icephobicity? Langmuir. 27(6), 3059-3066.

Kim, P., Wong, T.S., Alvarenga, J., et al., 2012. Liquid-infused nanostructured surfaces with extreme anti-ice and anti-frost performance. ACS Nano. 6(8), 6569-6577.

Kulinich, S.A., Farhadi, S., Nose, K., et al., 2011. Superhydrophobic surfaces: are they really ice-repellent. Langmuir. 227, 25-29.

Lv, J.Y., Song, Y.L., Jiang, L., et al., 2014. Bio-inspired strategies for anti-icing. ACS Nano. 8(4), 3152-3169.

Liu, B., Zhang, K.Q., Tao, C., et al., 2016. Strategies of anti-icing: low surface energy or liquid-infused?. RSC. Adv. 6(74), 70251-70260.

Lazauskas, A., Guobience, A., Prosyčevas, I., et al., 2013. Water droplet behavior on superhydrophobic $\mathrm{SiO}_{2}$ nanocomposite films during icing/deicing cycles. Mater. Charact. 82, 9-16.

Mobarakeh, L.F., Jafari, R., Faraneh, M., 2013. The ice repellency of plasma polymerized hexamethyldisiloxane coating. Appl. Surf. Sci. 284, 459-463.

Mahadik, S.A., Fernando, P.D., Hegade, N.D., et al., 2013. Durability and restoring of 
superhydrophobic properties in silica-based coatings. J. Colloid Interface Sci. 405, 262-268.

McDonald, B., Patel, P., Zhao, B. X., 2017. Droplet freezing and ice adhesion strength measurement on super-cooled hydrophobic surfaces. J. Adhes. 93(5), 375-388.

Nosonovskv, M., Hejazi, V., 2012. Why superhydrophobic surfaces are not always icephobic. ACS Nano. 6, 8488-8491.

Ozbay, S., Yuceel, C., Erbil, H.Y., 2015. Improved icephobic properties on surfaces with a hydrophobic lubricating liquid. ACS Appl. Mater. Interfaces. 7(39), 2206722077.

Ozbay, S., Erbil, H.Y., 2016. Ice accretion by spraying supercooled droplets is not dependent on wettability and surface free energy of substrates. Colloid Surf. APhysicochem. Eng. 504, 210-218.

Oberli, L., Caruso, D., Hall, C, 2014. Condensation and freezing of droplets on superhydrophobic surfaces. Adv. Colloid. Interface. Sci. 210, 47-57.

Pérez, J.M.P., Márquez, F.P.G., Hernández, D.R., 2016. Economic viability analysis for icing blades detection in wind turbines. J. Clean Prod. 135, 1150-1160.

Parent, O., Ilinca, A., 2011. Anti-icing and de-icing techniques for wind turbines: ccritical review. Cold Reg. Sci. Tech. 65, 88-96.

Ruan, W., Wang, J.W., Liu, L.Q., et al., 2016. Superhydrophobic and anti-icing properties of sol-gel prepared alumina coatings. Russ. J. Non-Ferrous Metals. $57(6), 638-645$.

Rashid, T., Khawaja, H.A., Edvardsen, K., 2016. Review of marine icing and anti-/de- 
icing systems. J. Mar. Eng. Technol. 15(2), 79-87.

Shin, D.H., Shokuhfar, T., Choi, C.K., et al., 2011. Wettability changes of $\mathrm{TiO}_{2}$ nanotube surfaces. Nanotechnology. 22(31) (No. 315704).

Sultana, K. R., Dehghani, S. R., Pope, K., et al., 2018. A review of numerical modelling techniques for marine icing applications. Cold Reg. Sci. Tech. 145, 40-51.

Tavakoli, F., Kavehpour, H.P., 2015. Cold-induced spreading of water drops on superhydrophobic surfaces. Langmuir. 31(7), 2120-2126.

Wang, S.D., Jiang, Y.S., 2015. The durability of superhydrophobic films. Appl. Surf. Sci. 357, 1647-1657.

Zhang, P., Lv, F.Y., 2015. A review of the recent advances in superhydrophobic surfaces and the emerging energy-related applications. Energy. 82, 1068-1087.

Zhu, X.T., Zhang, Z.Z., Ge, B., et al., 2014. Fabrication of superhydrophobic carbon nanotube coating with good reusability and easy repairability. Colloid Surf. Aphysicochem. Eng. Asp. 444, 252-256.

Zhang, Y., Anim-Danso, E., Bekele, S., et al., 2016. Effect of surface energy on freezing temperature of water. ACS Appl. Mater. Interfaces. 8(27), 17583-17590. 